\section{3-hydroxi-anthranilic acid is early expressed in stroke}

\author{
A. Mangas, ${ }^{1,2,3}$ J. Yajeya, ${ }^{4}$ N. González, ${ }^{1,2}$ \\ I. Ruiz, ${ }^{1}$ M. Geffard, ${ }^{1,2}$ R. Coveñas ${ }^{3}$ \\ ${ }^{1}$ Gemacbio, Saint-Jean-d'Illac, France \\ ${ }^{2}$ Institute for the Development of \\ Research in Human Pathology and \\ Therapeutic (IDRPHT), Talence, France \\ IInstitute of Neurosciences of Castilla y \\ León (INCYL), Laboratory of \\ Neuroanatomy of the Peptidergic \\ Systems, University of Salamanca, Spain \\ ${ }^{4}$ School of Medicine, Department of \\ Physiology, University of Salamanca, \\ Spain
}

\section{Abstract}

Using an immunohistochemical technique, we have studied the distribution of $3-0 \mathrm{H}$ anthranilic acid (3-HAA) in the rat brain. Our study was carried out in control animals and in rats in which a stroke model (single transient middle cerebral artery occlusion) was performed. A monoclonal antibody directed against 3-HAA was also developed. 3-HAA was exclusively observed in the infarcted regions (ipsilateral striatum/cerebral cortex), 2, 5 and 21 days after the induction of stroke. In control rats and in the contralateral side of the stroke animals, no immunoreactivity for 3HAA was visualized. Under pathological conditions (from early phases of stroke), we reported for the first time the presence of 3-HAA in the mammalian brain. By double immunohistochemistry, the coexistence of 3-HAA and GFAP was observed in astrocytes. The distribution of 3-HAA matched perfectly with the infarcted regions. Our findings suggest that, in stroke, 3-HAA could be involved in the tissue damage observed in the infarcted regions, since it is well known that 3-HAA exerts cytotoxic effects.

\section{Introduction}

It has been reported that one of six men and one of five women (55-75 years old) will suffer from stroke sometime during their lives. Three phases have been described in stroke: excitotoxicity, inflammatory activation/apoptosis and regeneration. ${ }^{1}$ In stroke, an activation of caspases, cytokines and both nitric oxide (NO) and indoleamine 2, 3-dioxygenase (ID0) pathways occurs. In the latter pathway, nicotinamide adenine dinucleotide (NAD) and several intermediate metabolites (e.g., 3OH-anthranilic acid - 3-HAA) are generated. It is known that, at pathophysiological concentrations, some of these intermediate metabolites (e.g., 3-HAA) exert a cytotoxic effect ${ }^{2-4}$ and that, in the cerebrospinal fluid of patients suffering from Huntington's disease, the level of 3-HAA is increased. ${ }^{2,5}$ Thus, for example, it has been reported that 3 -HAA generates hydrogen peroxide and superoxide promoting oxidative protein damage, induces apoptosis, and inhibits the mitochondrial respiratory chain. ${ }^{3,6-11}$ Moreover, 3-HAA has been involved in the initiation, development and amplification of neurodegenerative processes. ${ }^{2}$ However, the distribution of immunoreactive structures containing 3-HAA in the mammalian central nervous system is currently unknown (in both normal and pathological conditions). In order to develop new strategies for the treatment of several pathologies (e.g., stroke, Huntington's disease), immunohistochemical studies should be carried out to know the distribution of immunoreactive structures containing 3 -HAA in the mammalian brain.

In light of the above, our first aim in this study was to develop a highly specific monoclonal antibody directed against 3-HAA, and the second to know the distribution of the immunoreactive structures containing 3-HAA in the brain of normal rats and in animals in which a stroke model (single transient middle cerebral artery occlusion) was induced.

\section{Materials and Methods}

\section{Single transient middle cerebral artery occlusion (tMCAO)}

This study was approved by the research commission of the University of Salamanca (Spain). The experimental procedure of this work was performed under the guidelines of the legal and ethics recommendations of French, Spanish and European laws. Eighteen adult male Wistar rats (350 g) were used. Animals were deeply anesthetized with isoflurane and, as previously described, the tMCAO chirurgical procedure was performed:13-15 using a rounded tip monofilament (Doccol Co., Sharon, MA, USA), a single transient middle artery cerebral occlusion was carried out for 50 min. tMCAO rats were divided into three groups: 2D, 5D and 21D. That is, animals were perfused two (2D), five (5D) or twenty-one (21D) days after the middle artery cerebral occlusion. In each experimental group, 6 animals were used: four tMCAO animals and two control rats.
Correspondence: Dr. Arturo Mangas, Institute of Neurosciences of Castilla y León (INCYL), Laboratory of Neuroanatomy of the Peptidergic Systems (Lab. 14), University of Salamanca, c/ Pintor Fernando Gallego 1, 37007 Salamanca, Spain.

Tel. + +34.923.294500 ext. 5315 -Fax: +34.923 .294549 . E-mail: mangasam@usal.es

Key words: Astrocyte; ischemia; glia; immunohistochemistry; monoclonal antibody; neurotoxicity; 3-0H-anthranilic acid.

Acknowledgments: this work has been supported by the European Union FP7 Collaborative Grant TargetBraIn (number 279017), Gemacbio S.A. Laboratories (Saint Jean d'lllac, France), and by the IDRPHT (Talence, France). We wish to thank Professors Miguel Merchán, Margarita Heredia and Adelaida Riolobos (INCYL, Salamanca, Spain) for technical facilities, Miss Marianny Pernía for technical assistance and Dr. Denis Bedat (CIHS, Encinitas, CA, USA) for supervising the English text.

Received for publication: 1 August 2016. Accepted for publication: 27 November 2016

This work is licensed under a Creative Commons Attribution-NonCommercial 4.0 International License (CC BY-NC 4.0).

(C) Copyright A. Mangas et al., 2016

Licensee PAGEPress, Italy

European Journal of Histochemistry 2016; 60:2709 doi:10.4081/ejh.2016.2709

\section{Antibody development}

As previously described, ${ }^{16}$ a primary antiserum was developed in BALB/c mice after immunization with 3 -HAA-bovine serum albumin (BSA) immunogen linked via ethylchloroformiate (ECF). Thus, $20 \mathrm{mg}$ of BSA were dissolved in water, whereas $10 \mathrm{mg}$ of 3-HAA were dissolved in methanol; later, $40 \mu \mathrm{L}$ of triethylamine were added to both solutions. Then, $375 \mu \mathrm{L}$ of dimethylformamide mixed with 25 $\mu \mathrm{L}$ of ECF was added in order to activate the 3HAA solution. After 10 min of activation, the 3 HAA solution was added into a tube containing the BSA solution. Using dialysis membranes (with cut-off limits between 12 and $16 \mathrm{KDa}$ ), the obtained conjugate (3-HAA-BSA) was purified by dialysis. Later, the purification was carried out as previously described. ${ }^{16}$

Once the 3-HAA-BSA was synthesized, mice were immunized with the immunogen (containing 3-HAA-BSA). Each immunization was carried out by administering $50 \mu \mathrm{L}$ of an immunogenic $\mathrm{NaCl}$ solution and $50 \mu \mathrm{L}$ of complete (only used in the first immunization) or incomplete Freund adjuvant. As previously described, ${ }^{16-18}$ serum samples were collected 
and the antisera were pre-purified by immunoabsorption and tested by ELISA. Once a highly specific polyclonal antibody was obtained, the fusion of SP2/0/Ag myeloma cells and mice splenocytes was performed and the screening and the selection of specific clones were carried out. Once the highly specific monoclonal antibody against 3 -HAA was obtained, cells were expanded in plastic flasks. Supernatant was collected every week, centrifuged and pre-purified with a saturated $\left(\mathrm{NH}_{4}\right)_{2} \mathrm{SO}_{4}$ solution, dialyzed in PBS and purified in an HiTrap protein G HP column (170404-01, GE Healthcare, Little Chalfont, UK). An Isotyping kit was used to determinate the type of immunoglobulin and chain (26179, ThermoScientific, Waltham, MA, USA): the anti-3-HAA antibody was characterized as an isotype Ig $G_{1}$ and $\kappa$ chain. The affinity estimated of the monoclonal anti-3-HAA antibody was $10^{-9} \mathrm{M}$ and its specificity was considered excellent because close molecules were not recognized by the antibody (Table 1).

\section{Immunohistochemical study}

Once the tMCAO model was carried out (2, 5 or 21 days), the immunocytochemical study was performed. As previously reported, ${ }^{16,17}$ animals were anaesthetized with isoflurane (4\% induction, 2.5\% maintenance) and perfused (4\% paraformaldehyde); the brains were dissected out, post-fixed in paraformaldehyde (4\%) $(15-18 \mathrm{~h})$ and cryoprotected in increasing sucrose baths (5-30\%) (5-7 days). Using a freezing microtome, $40 \mu$-thick brain sections were obtained and processed for immunohistochemistry. Sections were treated with $\mathrm{H}_{2} \mathrm{O}_{2}$ $(10 \mathrm{~mL})$ and methanol $(20 \mathrm{~mL})$ for $30 \mathrm{~min}$ to avoid possible interference by endogenous peroxidase. Then, sections were washed in PBS (30 min) and pre-incubated (30 min) in the mix solution (PBS containing $0.3 \%$ Triton $\mathrm{X}-100$ and $1 \%$ normal horse serum). Later, sections were incubated overnight at $4^{\circ} \mathrm{C}$ in the mix solution containing the monoclonal anti3 -HAA antibody (diluted 1/1,000, Gemacbio, Saint-Jean-d'Illac, France), the polyclonal goat anti-ionized calcium-binding adapter molecule 1 (IBA-1) antibody (1/1,500, Abcam), the polyclonal rabbit anti-glial fibrillary acidic protein (GFAP) antibody (1/100, Dako, Glostrup, Denmark) or the monoclonal anti-GFAP antibody (1/400, Abcam, Cambridge, UK). Sections were washed in PBS (30 min) and incubated at room temperature for $1 \mathrm{~h}$ with biotinylated anti-mouse/rabbit/goat immunogammaglobulin (BA-1,000; BA-9,200; BA-5,000, Vector Labs, Burlingame, CA, USA), diluted $1 / 200$ in the mix solution. After a rinse in PBS (30 min), sections were incubated (room temperature, 1 h) with the avidin-biotin-peroxidase complex (ABC, Vectastain PK-6,100) (1/100) and were washed in PBS (30 min) and Tris-HCl buffer
(15 min). Finally, the tissue-bound peroxidase was developed with $\mathrm{H}_{2} \mathrm{O}_{2}$, using 3,3 , diaminobenzidine as chromogen (5-10 min). Histological controls were carried out to confirm the specificity of the immunoreactivity: i) pre-absorption of the anti-3-HAA antibody with an excess $(100 \mathrm{~g} / \mathrm{mL})$ of 3 -HAA; ii) omission of the primary and/or secondary antibodies. No residual immunoreactivity was found in either case.

According to a previous published protocol, ${ }^{19} 3$-HAA immunoreactive sections (3-HAA was detected by the DAB developing procedure) were prepared for double-labelling immunohistochemistry (GFAP: 1/100, Dako). The chromogen 4-chloro-1-naphthol was used to reveal the second immunohistochemical reaction (GFAP). The brown product (3-HAA) of DAB is easily distinguishable from the 4chloro-1-naphthol which provides a blue precipitate (GFAP). ${ }^{19}$ The DAB reaction product masks the antigen and catalytic sites of the first sequence of immunoreagents, preventing an interaction of the second sequence with the reagents. ${ }^{20}$

The stereotaxic atlas of Paxinos and Watson ${ }^{21}$ was used for mapping and nomenclature. Photomicrographs were obtained with an Olympus DP50 digital camera attached to a Kyowa Unilux-12 microscope. Using a Leica DMRB photomicroscope/Neurolucida system (8.0; Microbrightfield-bioscience, Williston, VT, USA) photographs at low-power magnification were also obtained. To improve the visualization of results, only the brightness and contrast of the images were adjusted. Adobe Photoshop Elements software was used.

\section{Results}

The monoclonal anti-3-HAA antibody used here was fully characterized by ELISA (Table 1). This antibody showed an excellent specificity and a high affinity (the $\mathrm{IC}_{50}$ was $10^{-9} \mathrm{M}$ ). According to protocols previously described, ${ }^{16,17}$ the parameters studied in these assays were antibody titration, avidity and specificity versus other close structural analogues (Table 1). Competition experiments were carried out with different competitors (Table 1); these experiments were performed with the same amount of antibody (dilution: $1 / 30,000)$, but using different concentrations of the conjugated antigens (e.g., anthranilic acid) (Table 1). As previously reported, ${ }^{17}$ ELISA tests were performed with the dilution of $1 / 30,000$, corresponding to an optical density of 1 , at $492 \mathrm{~nm}$. The anti-3-HAA monoclonal antibody used in this study recognized, at very low concentrations, the specific target; this means that its avidity was very high for 3 HAA. ${ }^{17,18,22}$ No cross-reactivity between the target molecule (3-HAA) and the closest ana-

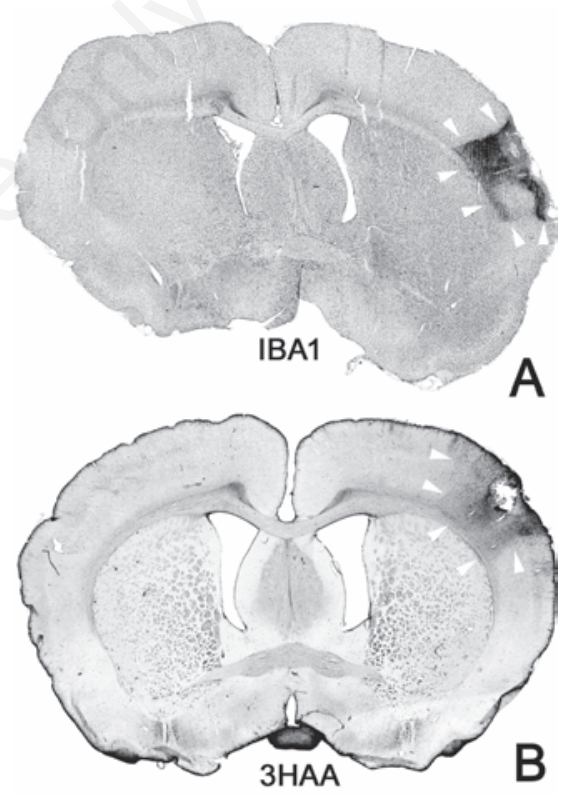

Figure 1. Low-power magnification (cerebral cortex: 21 days). Photographs were taken using a Leica microscope/ Neurolucida software. A) Section stained with IBA-1, a pan-microglia marker. B) Section stained with 3-HAA. Arrowheads indicate the infarcted region.

Table 1. Affinity and specificity of antibodies against conjugated 3-HAA.

\begin{tabular}{lc} 
Compounds & Cross-reactivity at half-displacement $\left(\mathrm{IC}_{50}\right)$ \\
3-hydroxy-anthranilic acid-BSA & 1 \\
Anthranilic acid-BSA & $1 />50,000$ \\
\hline Free anthranilic acid & $1 />50,000$ \\
Xanthurenic acid-BSA & $1 />50,000$ \\
\hline Picolinic acid-BSA & $1 />50,000$ \\
\hline
\end{tabular}

Using competition ELISA tests, cross-reactivity was calculated from the displacement curves at half-displacement: the best recognized conjugate was 3-HAA-BSA, whose concentration was divided by the concentration of each of the other conjugates. 
logues of 3-HAA was observed (Table 1).

In this study, three markers have been used: IBA-1 (to check that the tMCAO model was well performed; immunoreactivity was observed in microglia) (Figures 1A, $2 \mathrm{~A}, \mathrm{~B}$ and 3A), GFAP (to show astrocytes) (Figure $2 \mathrm{C}, \mathrm{D}$ ) and 3-HAA (Figures 1B, 2 E,F and 3 B-F). 3HAA was exclusively found in the infarcted regions (Figures 1B, 2 E,F and 3 B,C,E,F); that is, in the ipsilateral side (striatum and/or cerebral cortex) of animals in which the tMCAO model was carried out. The distribution of 3-HAA perfectly matches with the infarcted region (Figures $1 \mathrm{~B}$ and $3 \mathrm{~B}$ ). In this region, 3-HAA was observed in astrocytes, since cells containing 3-HAA or GFAP showed the same morphological characteristics (Figures $2 \mathrm{C}$-F and $3 \mathrm{C}, \mathrm{E}, \mathrm{F}$ ). In order to confirm this morphological observation, the coexistence of GFAP and 3-HAA was demonstrated by using a double-labelling immunohistochemistry technique: GFAP-immunoreactivity was observed in those astrocytes expressing 3-HAA (Figure $4 \mathrm{~A}$-F). In control animals, in the contralateral side of the TMCAO animals and in the non-infarcted region of the ipsilateral side, 3-HAA was not observed (Figures 1B and 3D), but as expected in these animals/areas microglia containing IBA-1 and GFAP-immunoreactive astrocytes were found. In the three experimental groups studied (2D, $5 \mathrm{D}, 21 \mathrm{D})$, all the findings mentioned above, were similar.

\section{Discussion}

We have demonstrated here, for the first time, the presence of 3-HAA (under pathological conditions) in the mammalian brain. In stroke, the expression of 3-HAA occurs from early stages and it is exclusively observed in the infarcted regions: there is a perfect anatomical relationship between the expression of 3-HAA and the infarcted region.

Regarding the development of the monoclonal anti-3-HAA antibody used here, it is very important during the process to maintain the original chemical structure/spatial conformation of the target. ${ }^{17,18}$ In this study, 3-HAA and the competitors used (e.g., anthranilic acid) were linked to BSA via different coupling agents (e.g., glutaraldehyde, ECF). To confirm that the original chemical structure of both target (3-HAA) and competitors was not modified by the linkage procedure, competition ELISA tests were carried out. These tests confirmed that the monoclonal anti-3-HAA antibody recognized specifically 3 -HAA, independently of the linkage procedure followed. ${ }^{18}$ Moreover, the monoclonal antibody did not recognize any competitor. The procedure pre- viously described is crucial for later studies (immunohistochemistry, Western-blotting) in order to recognize specifically the desired target and to avoid unspecific labelling (background). Due to the high affinity $\left(10^{-9} \mathrm{M}\right)$ and specificity of the monoclonal anti-3-HAA antibody used here, we have visualized for the first time the presence of 3-HAA in mammalian astrocytes. Moreover, the specificity of the immunoreactivity was confirmed when several histological controls (e.g., omission of the first antibody) were carried out.

In the tMCAO model, a considerable variability in the extension of the infarcted region has been reported..$^{23}$ It is also known that IBA-1 is specifically expressed in microglia and is up-regulated following cerebral ischemia. ${ }^{24}$ Here, in tMCAO animals, the up-regulation of IBA-1 was observed in the infarcted regions (striatum and/or cerebral cortex) and hence, in some animals, the location and/or extension of the infarcted regions were different. Since astrocytes containing 3 -HAA were exclusively observed in the infarcted regions, the location and/or extension of the regions showing 3-HAA-immunoreactive structures (striatum and/or cerebral cortex) were different in some tMCAO animals. In sum, our data are agreement with the observations previously reported in the tMCAO experimental model, ${ }^{23,24}$ confirming that the chirurgical procedure (tMCA0) was correctly carried out.
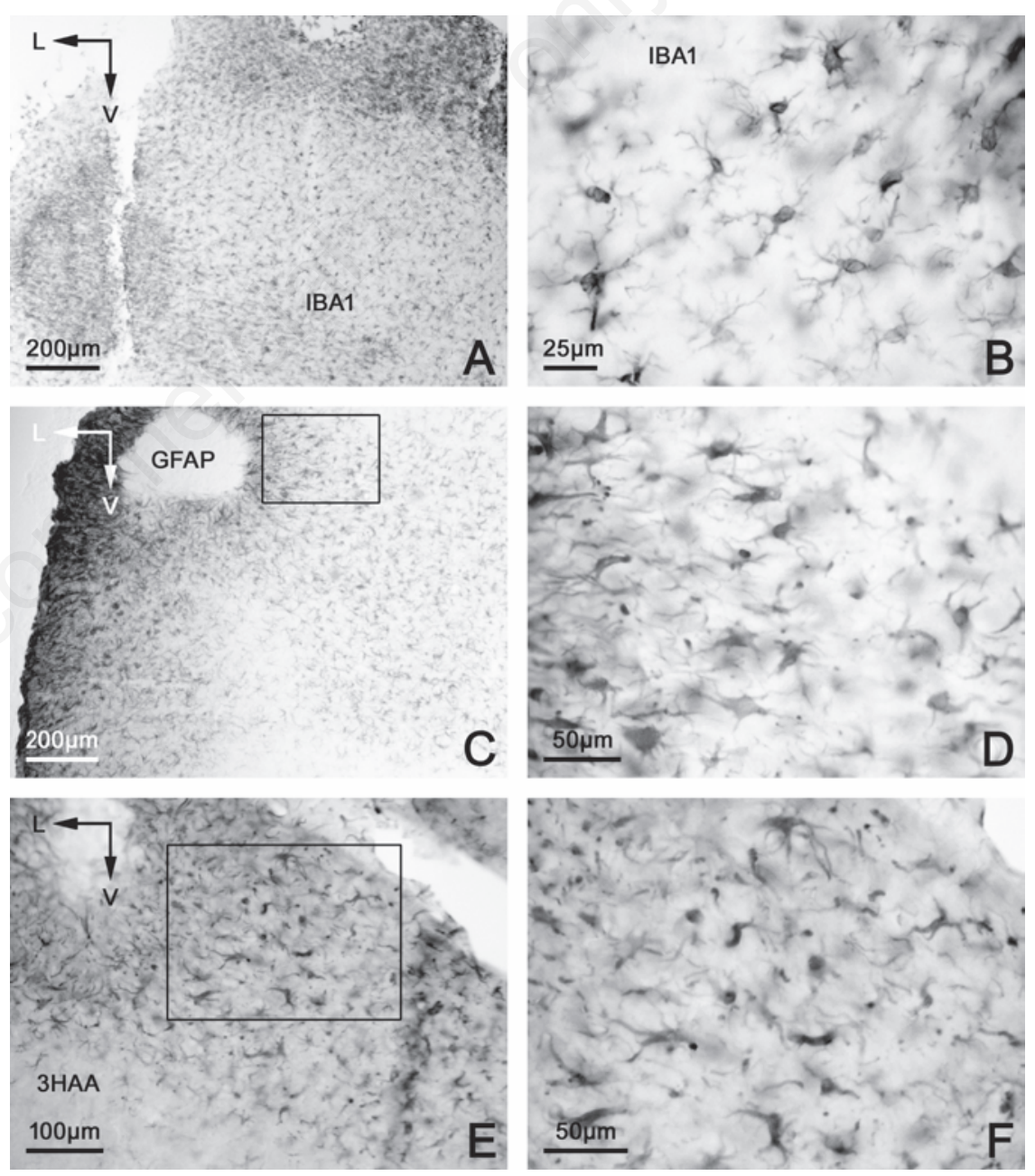

Figure 2. A) Infarcted region (ipsilateral side; cerebral cortex: 21 days) stained with antiIBA-1. B) Infarcted region; microglia containing IBA-1. C) Infarcted region; coronal contiguous section to that shown in Figure 1A. D) Higher-power magnification of the rectangle showed in Figure 1C; GFAP-immunoreactive astrocytes. E) Infarcted region; coronal contiguous section to that shown in Figure 1C; 3-HAA is observed in astrocytes. F) Higher-power magnification of the region delimited by a rectangle in Figure 1E. $\mathrm{L}$, lateral; $\mathrm{V}$, ventral. 
We have demonstrated the coexistence of 3 HAA and GFAP in astrocytes. This was exclusively observed in those astrocytes located in the infarcted region. In our study, two different anti-GFAP antibodies were used and, in both cases, the results were identical. It is important to note that the expression of 3 -HAA was observed in the three experimental groups studied (2D, 5D, 21D); this means that, in stroke, 3-HAA was expressed from early stages and that this expression continued 21 days post-stroke.

In the ID0 pathway, several important processes occur: tryptophan is catabolised and intermediate metabolites (e.g., 3-HAA, kynurenine, quinolinic acid, kynurenic acid) and NAD are generated. This pathway regulates the immune response (e.g., 3-HAA controls the population of Th 17 lymphocytes) ${ }^{25-27}$ and is altered in several pathologies (e.g., stroke, Alzheimer's disease, amyotrophic lateral sclerosis, depression, Huntington's disease, multiple sclerosis, Parkinson's disease, schizophrenia). ${ }^{3,6,28,29}$ It is known that the metabolites generated in the ID0 pathway exert opposite effects: kynurenic acid has a neuroprotective action, whereas 3-HAA (a free radical generator), quinolinic, and picolinic acids exert a cytotoxic effect. ${ }^{6,29-33}$ In the case of 3-HAA, it is well known that this intermediate metabolite and precursor of quinolinic impairs brain energy metabolism, generates hydrogen peroxide and superoxide promoting oxidative protein damage and induces apoptosis., ${ }^{36-11}$ It has been also reported that a neurotoxic effect was observed when the concentration of 3-HAA was >100 nM, but a lower concentration increased the level of $\mathrm{NAD}^{+}$, exerting a beneficial effect on cultured human neurons and astrocytes. ${ }^{2,34}$ The former is in agreement with our anatomical results in which an overexpression of 3-HAA (observed from early stages in stroke) was exclusively found in the infarcted regions. Moreover, in stroke, a down-regulation of the expression of 3-HAA has been observed (personal communication, unpublished data) after the administration of new drug candidates exerting anti-oxidative and anti-inflammatory effects. ${ }^{35}$ Thus, 3 -HAA immunoreactivity was modulated by these drugs. This preliminary finding is in agreement with published data reporting that inflammatory mediators (e.g., lipopolysaccharide, interleukin-1) stimulates the kynureninase enzyme to produce 3 -HAA. ${ }^{36}$ According to the above mentioned data, it seems that in the tMCAO model, the ipsilateral overexpression of 3 -HAA is involved in the tissue damage observed in the infarcted regions. However, this must be confirmed in future studies. 3 -HAA is expressed from early stages in stroke and hence, in the future, strategies against the overexpression of 3-HAA (once its cytotoxic action was fully demonstrated) should be developed. It is known that two enzymes (microsomal hydroxylase, kynureninase) are involved in the synthesis of $3-\mathrm{HAA}^{37}$ and, according to our anatomical results, it seems that they could be up-regulated in astrocytes under ischemic conditions. Under these conditions, an increase in the level of 3-HAA will produce an increase in the level of quinolinic acid (this intermediate metabolite of the IDO pathway exerts the highest cytotoxic effect). ${ }^{12,38}$ Accordingly, it has been reported that quinolinic acid favored neuronal/glial damage and/or death by at least nine different processes and that inhibitors of the 3 -HAA oxidase (involved in the synthesis of quinolinic acid from 3 -HAA) reduced quinolinic acid accumulation and deficits caused. . $^{, 12,34,38-41}$

In conclusion, in a tMCA0 stroke model, i) for the first time, 3-HAA has been observed in the astrocytes of mammals; ii) 3-HAA was exclusively found in the infarcted regions; in control animals, 3-HAA-immunoreactive structures were not visualized; iii) from early stages, an overexpression of 3-HAA has been observed; iv) an anatomical relationship between the expression of 3 -HAA and the infarcted region occurred; and v) 3-HAA, acting as a possible cytotoxic factor, could be involved in the tissue damage observed in the infarcted regions.
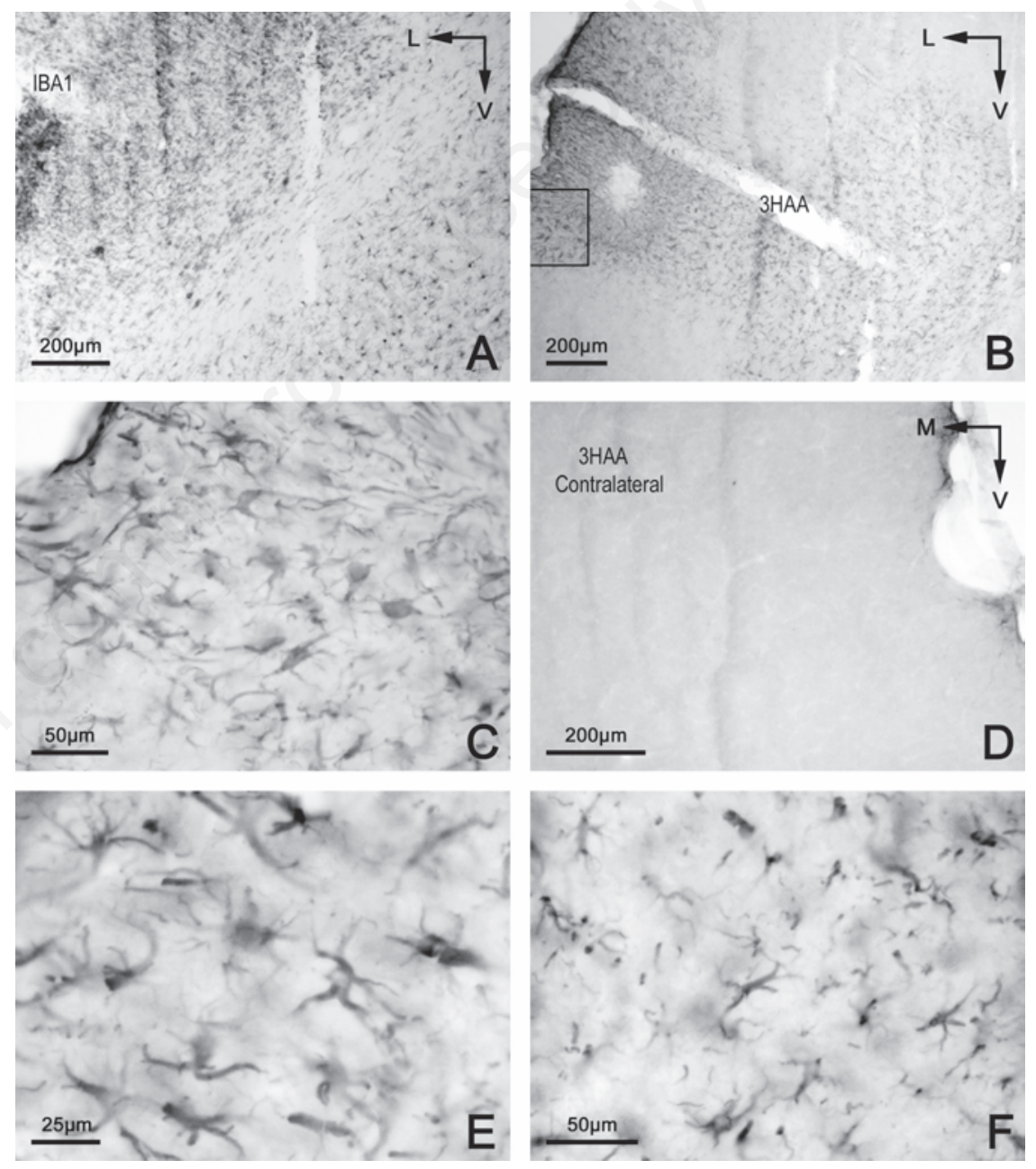

Figure 3. Infarcted region (ipsilateral side; cerebral cortex: 21 days) stained with antiIBA-1 (A) or anti-3-HAA (B). Photographs in Figure 2A and B were taken from contiguous sections. The border of the infarcted region is clearly observed in Figure 2B. 3-HAAimmunoreactive astrocytes are exclusively found in the infarcted region. C) Higher-power magnification of the region delimited by a rectangle in Figure 2B. D). Contralateral cerebral cortex of the same section shown in Figure 2B; note the absence of immunoreactivity for 3-HAA. E, F) High-power magnification of the infarcted region; 3-HAA-immunoreactive astrocytes can be observed. $L$, lateral; $M$, medial; $V$, ventral. 

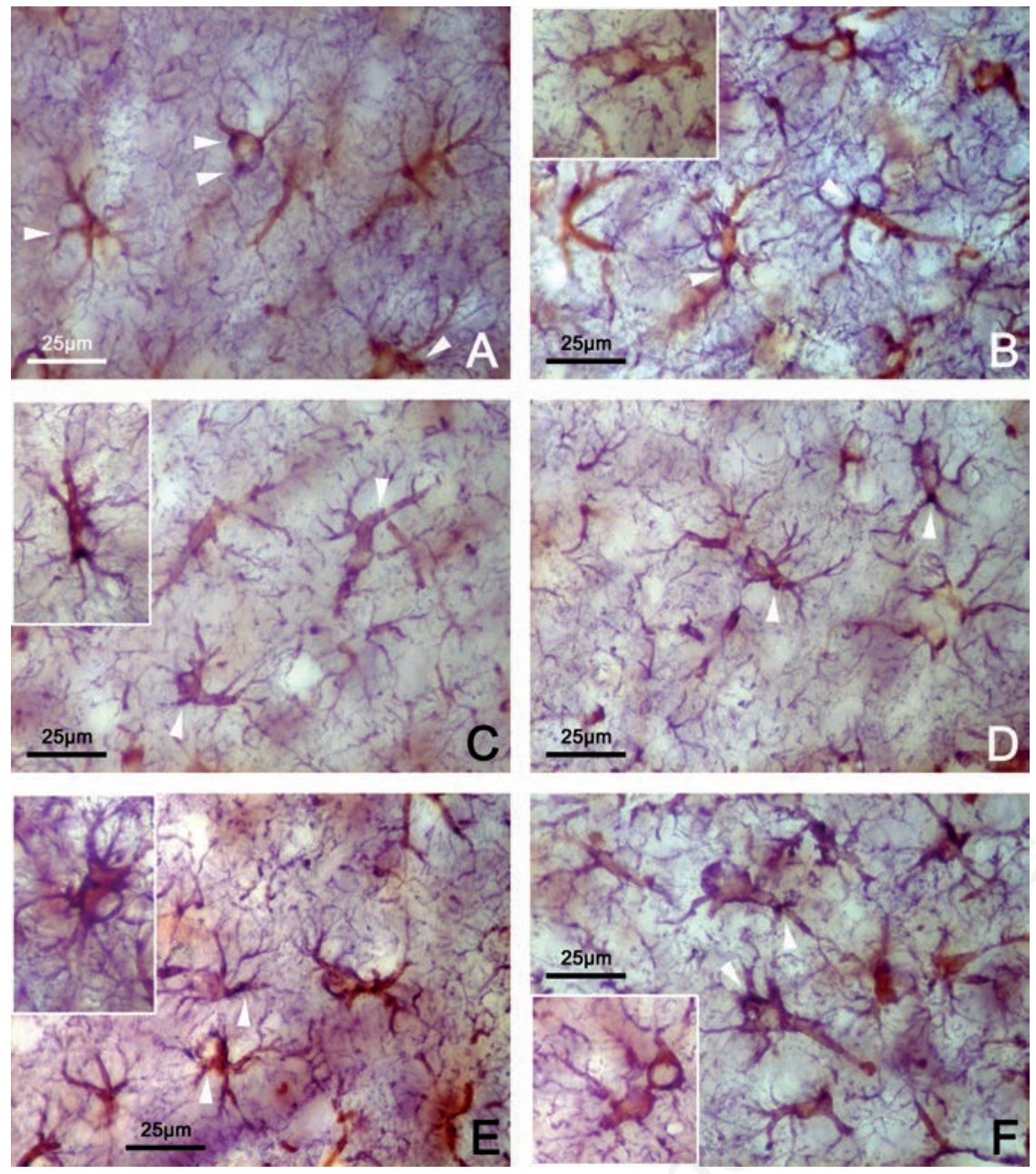

Figure 4. Infarcted region (ipsilateral side; cerebral cortex: 21 days); double-labeling immunohistochemistry. In astrocytes, the coexistence of GFAP (blue labelling, arrowheads) and 3-HAA (brown labelling) is observed.

\section{References}

1. Tatro JB. Melanocortins defend their territory: multifaceted neuroprotection in cerebral ischemia. Endocrinology 2006;147:1122-5.

2. Braidy N, Grant R, Brew BJ, Adams S, Jayasena T, Guillemin GJ. Effects of kynurenine pathway metabolites on intracellular $\mathrm{NAD}^{+}$synthesis and cell death in human primary astrocytes and neurons. Int J Tryptophan Res 2009;2:61-9.

3. Bohár Z, Toldi J, Fülöp F, Vécsei L. Changing the face of kynurenines and neurotoxicity: therapeutic considerations. Int J Mol Sci 2015;16:9772-93.

4. Pérez-De La Cruz V, Königsberg M, Santamaría A. Kynurenine pathway and disease: an overview. CNS Neurol Disord Drug Targets 2007;6:398-410.

5. Smith AJ, Stone TW, Smith RA. Neurotoxicity of tryptophan metabolites.
Biochem Soc Trans 2007;35:1287-9.

6. Goldstein LE, Leopold MC, Huang X, Atwood CS, Saunders AJ, Hartshorn M, et al. 3-Hydroxykynurenine and 3-hydroxyanthranilic acid generate hydrogen peroxide and promote alpha-crystalline cross-linking by metal ion reduction. Biochemistry 2000;39:7266-75.

7. Schwarcz R, Pellicciari R. Manipulation of brain kynurenines: glial targets, neuronal effects, and clinical opportunities. J Pharmacol Exp Ther 2002;303:1-10.

8. Dykens JA, Sullivan SG, Stern A. Oxidative reactivity of the tryptophan metabolites 3 hydroxyanthranilate, cinnabarinate, quinolinate and picolinate. Biochem Pharmacol 1987;36:211-7.

9. Schuck PF, Tonin A, da Costa Ferreira G, Viegas CM, Latini A, Duval Wannmacher $\mathrm{CM}$, et al. Kynurenines impair energy metabolism in rat cerebral cortex. Cell Mol Neurobiol 2007;27:147-60.

10. Quagliariello E, Papa S, Saccone C, Alifano
A. Effect of 3-hydroxyanthranilic acid on the mitochondrial respiratory system. Biochem J 1964;91:137-46.

11. Morita T, Saito K, Takemura M, Maekawa $\mathrm{N}$, Fujigaki S, Fujii H, et al. 3-hydroxyanthranilic acid, an L-tryptophanmetabolite, induces apoptosis in monocyte-derived cells stimulated by interferon-gamma. Ann Clin Biochem 2001;38:242-51.

12. Lim CK, Fernández-Gómez FJ, Braidy N, Estrada C, Costa C, Costa S, et al. Involvement of the kynurenine pathway in the pathogenesis of Parkinson's disease. Prog Neurobiol 2016 pii: S03010082(15)30055-1. doi: 10.1016/j.pneurobio.2015.12.009. [Epub ahead of print]

13. Longa EZ, Weinstein PR, Carlson S, Cummins R. Reversible middle cerebral artery occlusion without craniectomy in rats. Stroke 1989;20:84-91.

14. Qiao M, Zhao Z, Barber PA, Foniok T, Sun $\mathrm{S}$, Tuor UI. Development of a model of recurrent stroke consisting of a mild transient stroke followed by a second moderate stroke in rats. J Neurosci Methods 2009; 184:244-50.

15. Uluç K, Miranpuri A, Kujoth GC, Aktüre E, Ba kaya MK. Focal cerebral ischemia model by endovascular suture occlusion of the middle cerebral artery in the rat. J Vis Exp 2011;48:1978.

16. Mangas A, Bodet D, Duleu S, Yajeya J, Geffard M, Coveñas R. Direct visualization of retinoic acid in the rat hypothalamus: an immunohistochemical study. Neurosci Lett 2012;509:64-8.

17. Mangas A, Coveñas R, Bodet D, Geffard M, Aguilar LA, Yajeya J. Immunocytochemical visualization of D-glutamate in the rat brain. Neuroscience 2007;144:654-64.

18. Mangas A, Coveñas R, Geffard M. Antisera and immunocytochemical techniques. In: A. Mangas, R. Coveñas, M. Geffard (eds.) Brain molecules: from vitamins to molecules for axonal guidance. Trivandrum: Transworld Research Network; 2008. p. 1-26.

19. Marcos P, Arroyo-Jiménez MM, Lozano G, González-Fuentes J, Lagartos-Donate MJ, Coveñas R. Mapping of tyrosine hydroxylase in the diencephalon of alpaca (Lama pacos) and co-distribution with somatostatin-28 (1-12). J Chem Neuroanat 2013; 50-51:66-74.

20. Marcos P, Corio M, Dubourg P, Coveñas R, Tramu G. Double immunocytochemistry in pre-embedding electron microscopy for the detection of neurotensin and tyrosine hydroxylase in the guinea pig, using two primary antisera raised in the same species. Brain Res Brain Res Protoc 1997;2:1-8.

21. Paxinos G, Watson C. The rat brain in 
stereotaxic coordinates. Sydney: Academic Press; 1982.

22. Mangas A, Coveñas R, Geffard K, Geffard M, Marcos P, Insausti R, et al. Riboflavinlike inmunoreactive fibers in the monkey brain. Anat Embryol 2006;211: 67-72.

23 Lin X, Miao P, Wang J, Yuan F, Guan Y, Tang Y, et al. Surgery-related thrombosis critically affects the brain infarct volume in mice following transient middle cerebral artery occlusion. PLoS One 2013;8: e75561.

24. Ito D, Tanaka K, Suzuki S, Dembo T, Fukuuchi Y. Enhanced expression of Ibal, ionized calcium-binding adapter molecule 1, after transient focal cerebral ischemia in rat brain. Stroke 2001;32:1208-15.

25. Moffett JR, Namboodiri MA. Tryptophan and the immune response. Immunol. Cell Biol 2003;81:247-65.

26. Campbell BM, Charych E, Lee AW, Möller T. Kynurenines in CNS disease: regulation by inflammatory cytokines. Front Neurosci 2014;8:12.

27. YanY, Zhang GX, Gran B, Fallarino F, Yu S, $\mathrm{Li} \mathrm{H}$, et al. IDO upregulates regulatory T cells via tryptophan catabolite and suppresses encephalitogenic T cell responses in experimental autoimmune encephalomyelitis. J Immunol 2010;185: 5953-61.

28. Gold AB, Herrmann N, Swardfager W, Black SE, Aviv RI, Tennen G, et al. The relationship between indoleamine 2,3-dioxy- genase activity and post-stroke cognitive impairment. J Neuroinflamm 2011;8:17.

29. Lovelace MD, Varney B, Sundaram G, Lennon MJ, Lim CK, Jacobs K, et al. Recent evidence for an expanded role of the kynurenine pathway of tryptophan metabolism in neurological diseases. Neuropharmacology 2017;112(Pt B):37388 [Epub 2016 Mar 16].

30. Guillemin GJ, Smythe G, Takikawa 0, Brew BJ. Expression of indoleamine 2,3dioxygenase and production of quinolinic acid by human microglia, astrocytes, and neurons. Glia 2005;49:15-23.

31. Guillemin GJ, Cullen KM, Lim CK, Smythe GA, Garner B, Kapoor V, et al. Characterization of the kynurenine pathway in human neurons. J Neurosci 2007; 27:12884-92

32. Chen Y, Stankovic R, Cullen KM, Meininger V, Garner B, Coggan S, et al. The kynurenine pathway and inflammation in amyotrophic lateral sclerosis. Neurotox Res 2010; 8: 32-42.

33. Sundaram G, Brew BJ, Jones SP, Adams S, Lim CK, Guillemin GJ. Quinolinic acid toxicity on oligodendroglial cells: relevance for multiple sclerosis and therapeutic strategies. J Neuroinflammation 2014;11: 204.

34. Pérez-de la Cruz V, Amori L, Sathyasaikumar KV, Wang XD, Notarangelo FM, Wu HQ, et al. Enzymatic transamination of D-kynurenine generates kynurenic acid in rat and human brain. J Neurochem 2012;120:1026-35.

35. Mangas A, Coveñas R, Geffard M. New drug therapies for multiple sclerosis. Curr Opin Neurol 2010;23:287-92.

36. Campbell BM, Charych E, Lee AW, Möller T. Kynurenines in CNS disease: regulation by inflammatory cytokines. Front Neurosci 2014; 8:1-22.

37. Bender DA, McCreanor GM. The preferred route of kynurenine metabolism in the rat. Biochim Biophys Acta 1982;717:56-60.

38. Pérez-de la Cruz V, Carrillo-Mora P, Santamaría A. Quinolinic Acid, an endogenous molecule combining excitotoxicity, oxidative stress and other toxic mechanisms. Int J Tryptophan Res 2012;5:1-8.

39. Guillemin GJ. Quinolinic acid the inescapable neurotoxin. FEBS J 2012;279: 1356-65.

40. Lugo-Huitrón R, Blanco-Ayala T, UgaldeMuñiz P, Carrillo-Mora P, Pedraza-Chaverrí J, Silva-Adaya D, et al. On the antioxidant properties of kynurenic acid: free radical scavenging activity and inhibition of oxidative stress. Neurotoxicol Teratol 2011;33:538-47.

41. Blight AR, Cohen TI, Saito K, Heyes MP. Quinolinic acid accumulation and functional deficits following experimental spinal cord injury. Brain 1995;118:735-52. 